\title{
A New PLL Simulation Validation for Three-phase Grid under Heavy Distorted Conditions
}

\author{
http://dx.doi.org/10.3991/ijoe.v11i7.4765 \\ Yingwen Long ${ }^{1}$ and Yuhong Sun ${ }^{2}$ \\ ${ }^{1}$ Shanghai University of Engineering Science, Shanghai, China \\ ${ }^{2}$ Shanghai Surpass Sun Electric Co. Ltd, Shanghai, China
}

\begin{abstract}
The precision of phase-locked loop (PLL) has a direct effect on the output performance for three-phase grid-connected inverter or three-phase active PFC. In this paper, a new three-phase digital closed-loop phase-locked algorithm is proposed on the basis of synchronous reference frame transformation. Synchronous simulation of the PLL techniques is a good choice even if the polluted three-phase grid such as the harmonics, phase jump and unbalance. Finally, MATLAB digital simulation results prove that the proposed PLL can fast and accurately track the positive sequence fundamental components of three-phase grid voltage.
\end{abstract}

Index Terms-Phase-locked loop, Positive sequence, Synchronous reference frame

\section{INTRODUCTION}

With the development of economy, more and more non-linear loads are connected to the electric networks. Therefore, distortions and transients such as harmonics and phase shifts often occur to the grid [1, 2]. Moreover, it is reasonable to expect that the level of pollution will increase in the future. Obviously, the accurate phase angle information of utility voltage is very essential to grid-connected inverters or active PFC. Various synchronization techniques have been proposed in literatures. The zero crossing detection methods [3], the space-vector filtering (SVF) method [4], the artificial neural networks (ANN) method [5], the recursive weighted least-squares estimation (WLSE) algorithm [6], the discrete Fourier transform (DFT) and its modifications [7], the methods based on the concept of adaptive notch filtering (ANF) [8], the Kalman filtering technique [9], the frequency-locked loop (FLL) methods [10], three phase open-loop phase-locked method [11] and the phase-locked loop (PLL) algorithms $[12,13]$ are among the existing synchronization techniques.

This paper puts forward a new closed-loop phaselocked method. The positive sequence fundamental components of grid voltages are extracted with the help of an all-pass filter and a second-order low-pass filter. The proposed PLL demonstrates superior performances in terms of synchronization signals even under the conditions of harmonics, phase shifts and unbalance that exist in the utility voltages used as the basis of synchronization. In addition, the salient feature of the proposed PLL is the simplicity of structure which renders itself for digital implementation.

\section{CONVENTIONAL CLOSED-LOOP PHASE- LOCKED METHOD}

The basic structure of three-phase PLL based on synchronous reference frame transformation is shown in Fig.1.

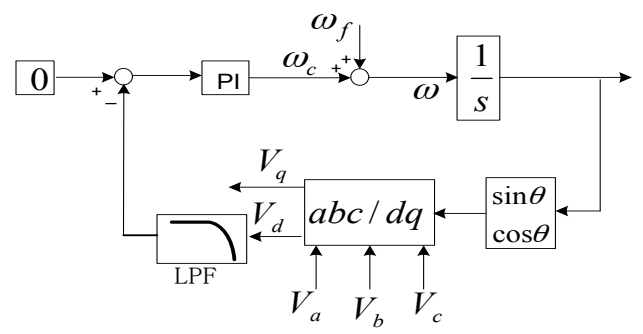

Fig. 1 Block diagram of three-phase PLL based on synchronous reference frame transformation

Where $V_{a}, V_{b}, V_{c}$ stand for three-phase utility voltage, $\sin \theta$ and $\cos \theta$ are the output of PLL. $\omega_{c}$ is the output of PI controller, $\omega_{f}$ is the feed-forward component of the grid angular frequency.

The three-phase polluted grid voltage can be written as:

$$
\left\{\begin{array}{l}
V_{a}=\sum_{n=1}^{\infty} V_{n+} \sin \left(n \omega t+\sigma_{n+}\right)+\sum_{n=1}^{\infty} V_{n-} \sin \left(n \omega t+\sigma_{n-}\right)+V_{0} \\
V_{b}=\sum_{n=1}^{\infty} V_{n+} \sin \left(n \omega t-\frac{2 \pi}{3}+\sigma_{n+}\right)+\sum_{n=1}^{\infty} V_{n-} \sin \left(n \omega t+\frac{2 \pi}{3}+\sigma_{n-}\right)+V_{0} \\
V_{c}=\sum_{n=1}^{\infty} V_{n+} \sin \left(n \omega t+\frac{2 \pi}{3}+\sigma_{n+}\right)+\sum_{n=1}^{\infty} V_{n-} \sin \left(n \omega t-\frac{2 \pi}{3}+\sigma_{n-}\right)+V_{0}
\end{array}\right.
$$

Where $V_{1+}, V_{1-}, V_{n+}, V_{n-}$ are the amplitude of fundamental positive sequence component, fundamental negative sequence component, n-order positive sequence component, n-order negative sequence component respectively. $\sigma_{1+}, \sigma_{1-}, \sigma_{n+}, \sigma_{n-}$ are the original phase of fundamental positive sequence component, fundamental negative sequence component, n-order sequence component, n-order negative sequence component respectively. $\omega$ is the fundamental angular frequency. $V_{0}$ is the zero sequence component.

With the help of two transformation matrixes, a threephase PLL control scheme can transform the three-phase 
grid voltage vectors into $\mathrm{d}-\mathrm{q}$ synchronous rotational coordinates:

$$
\left[\begin{array}{l}
V_{d} \\
V_{q}
\end{array}\right]=T_{a b c / \alpha \beta} * T_{\alpha \beta / d q}\left[\begin{array}{c}
V_{a} \\
V_{b} \\
V_{c}
\end{array}\right]
$$

Where $T_{a b c / \alpha \beta}$ and $T_{\alpha \beta / d q}$ are two transformation matrixes. $\hat{\omega}$ is the evaluated fundamental angular frequency.

Then the formula (2) can be rewritten as

$$
\left[\begin{array}{l}
V_{d} \\
V_{q}
\end{array}\right]=\frac{3}{2}\left[\begin{array}{l}
\sum_{n=1}^{\infty} V_{n+} \sin \left((n \omega-\hat{\omega}) t+\sigma_{n+}\right)+\sum_{n=1}^{\infty} V_{n-} \sin \left((n \omega+\hat{\omega}) t+\sigma_{n-}\right) \\
\sum_{n=1}^{\infty}-V_{n+} \cos \left((n \omega-\hat{\omega}) t+\sigma_{n+}\right)+\sum_{n=1}^{\infty} V_{n-} \cos \left((n \omega+\hat{\omega}) t+\sigma_{n-}\right)
\end{array}\right] .
$$

If the three-phase grid voltage contains little harmonic component and the evaluated value $\hat{\omega} t$ is close to $\omega t$, $V_{d}$ can be simplified as

$$
V_{d}=\frac{3}{2} V_{1+} \sin (\omega t-\hat{\omega} t) \approx \frac{3}{2} V_{1+}(\omega t-\hat{\omega} t)
$$

A satisfactory closed-loop system should provide both fast tracking ability and high phase-locked accuracy. Conventional three-phase PLL systems use low-pass filters (LPF) to reduce the impact of harmonics after the extraction of d-axis component. Lower cut-off frequency of the low-pass filter causes lesser distortions in the estimated phase angle. However, this results in a poor dynamic performance. So, a trade-off should be made between the two features. Furthermore, if the three-phase utility voltage is seriously polluted, it will be impossible to acquire the accurate phase information of the positive sequence utility voltage at fundamental frequency only depending on the regulation of cut-off frequency. Thus, the three-phase closed-loop system should be redesigned in consideration of heavy pollutions such as harmonics, frequency variations, phase shifts and unbalance that usually exist in the utility voltages.

\section{NEW THREE-PHASE CLOSED-LOOP PHASE- LOCKED METHOD}

Based on the theory of symmetrical components, the positive sequence components of three-phase utility voltages $\left[\begin{array}{lll}V_{a}^{+} & V_{b}^{+} & V_{c}^{+}\end{array}\right]$can be obtained as

$$
\left[\begin{array}{c}
V_{a}^{+} \\
V_{b}^{+} \\
V_{c}^{+}
\end{array}\right]=\left[\begin{array}{c}
\frac{1}{2} V_{a}-\frac{1}{2 \sqrt{3} j}\left(V_{b}-V_{c}\right) \\
-\left(V_{a}^{+}+V_{c}^{+}\right) \\
\frac{1}{2} V_{c}-\frac{1}{2 \sqrt{3} j}\left(V_{a}-V_{b}\right)
\end{array}\right]
$$

For the formula (5), the operator $-j \frac{\pi}{2}$ can be implemented with a full-pass filter which is designed to provide unit gain and realize $-90^{\circ}$ phase shift at the fundamental frequency. The harmonics in three-phase utility voltages would pollute the synchronization signals, this paper considers the second-order low-pass filter as shown in the formula (6) to reduce the harmonics and implement the $-90^{\circ}$ phase shift at the fundamental frequency without any distortion as required to obtain the positive sequence components and eliminate harmonics synchronously.

The specified transfer function of the second-order LPF in the Laplace domain is expressed as

$$
L P F=\frac{\omega_{n}^{2}}{s^{2}+\omega_{n} s+\omega_{n}^{2}}
$$

Where $\omega_{n}$ is the natural angular frequency that should be equal to the fundamental angular frequency.

The Bode plot of the filter is shown in Fig.2. It is obvious to see that the filter shows itself as unit gain and $-90^{\circ}$ phase shift at $50 \mathrm{~Hz}$. In addition, high frequency utility voltage harmonics are significantly reduced. For instance, the second harmonic is attenuated to $-11.2 \mathrm{~dB}$, the third harmonic, $-18.7 \mathrm{~dB}$ and the fifth harmonic, -27.8 $\mathrm{dB}$ at the output of the second-order LPF.

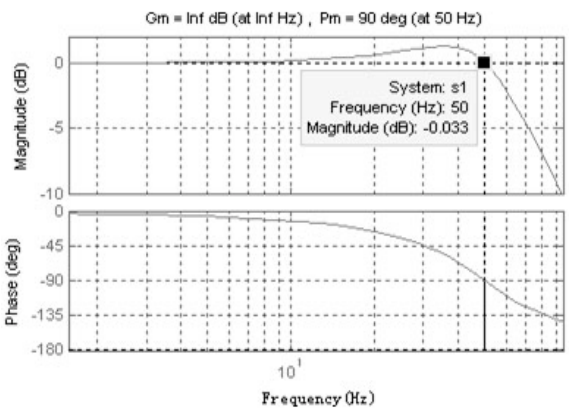

Fig. 2 Bode plot of the second-order LPF

The proposed three-phase PLL structure is shown in Fig.3. The polluted three-phase utility voltage is transformed into $\alpha-\beta$ stationary coordinates. In this way, the feedback loop acquires the positive sequence components and attenuates the high-frequency harmonics synchronously. In addition, the conventional integral is replaced by a sine table ranging from $0^{0}$ to $360^{\circ}$ which makes the proposed synchronization method more convenient for digital implementation.

By means of a transfer coefficient, that is $k$, the estimated angular frequency is transformed into the index value of the sine table listed in digital program. The transfer coefficient is defined as:

$$
k=\frac{180}{\pi} * \frac{N}{360} * T_{s}
$$


Where $\mathrm{N}$ is the number of specified sine values in the sine table, and $T_{s}$ is the sampling period.

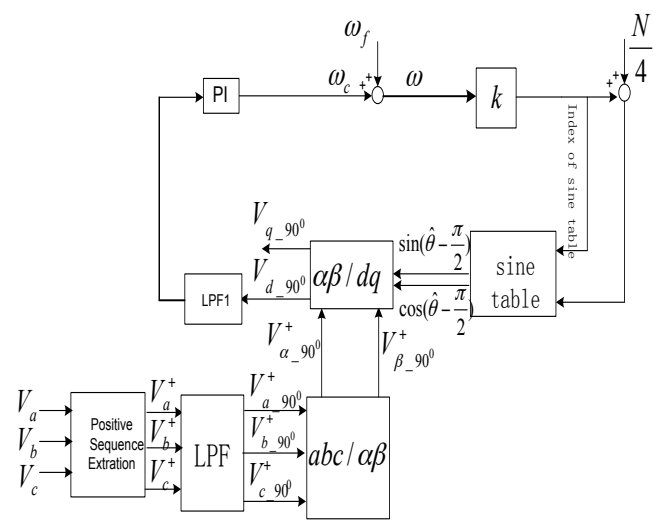

Fig. 3 Block diagram of the new three-phase digital PLL

\section{SIMULATION}

Performance of the proposed PLL method is evaluated by means of a number of simulations.

The full-pass filter $\left(\omega_{n}-s\right) /\left(s+\omega_{n}\right)$ and the secondorder LPF $\omega_{n}^{2} /\left(s^{2}+\omega_{n} s+\omega_{n}^{2}\right)$ are changed into discrete model in order to implement the digital control. Eventually, the hybrid simulation of the digital PLL scheme is realized by $\mathrm{C}$ language and Simulink module in Matlab. The key PLL parameters are shown as

$$
T_{s}=50 * 10^{-6} s, N=720, k=0.00573, L P F 1=\frac{850}{s+850}
$$

To show the promising behaviors of the proposed synchronization method, three operation conditions are considered in the following simulations.

\section{A. Harmonic distortion Simulation}

Usually, the odd harmonic contents in the utility voltage are high, while even harmonics are few and can be neglected. Hereby, the three-phase utility voltages in the presence of heavy harmonic pollution are shown as:

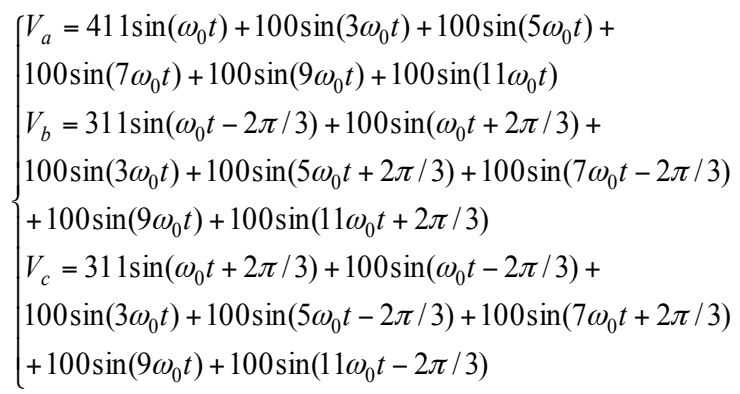

The fundamental frequency of utility voltage is $50 \mathrm{~Hz}$. The THD of output signal of the proposed PLL is shown in Fig.4. Fig.5 shows dynamic response of the proposed PLL, the three waveforms imply the amplified sinusoidal synchronization signal, the A-phase positive sequence fundamental voltage and the A-phase polluted utility voltage.
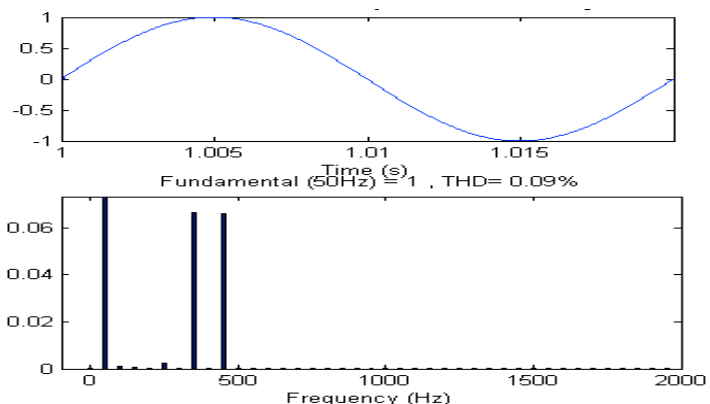

Fig.4 Phase-locked precision of the proposed PLL

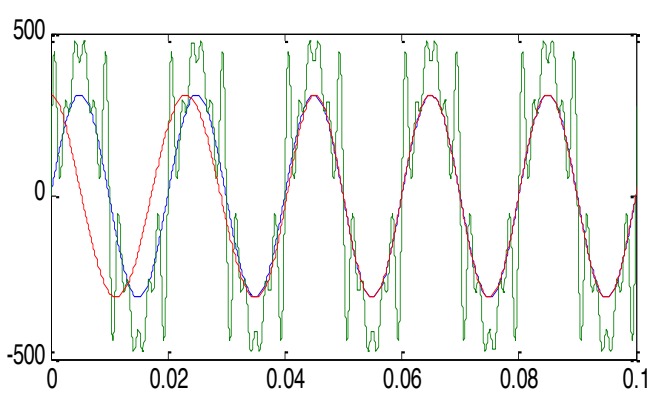

Fig.5 Dynamic response of the proposed PLL

\section{B. Three-Phase Voltage Phase Jumping Simulation}

Before 0.1 second, the three-phase utility voltages are still shown as the formula (8). After 0.1 second, the threephase utility voltages happen to shift. The results are shown as the formula (9). At the 0.1 second, the positive fundamental phase of the three-phase grid change by $180^{\circ}$.

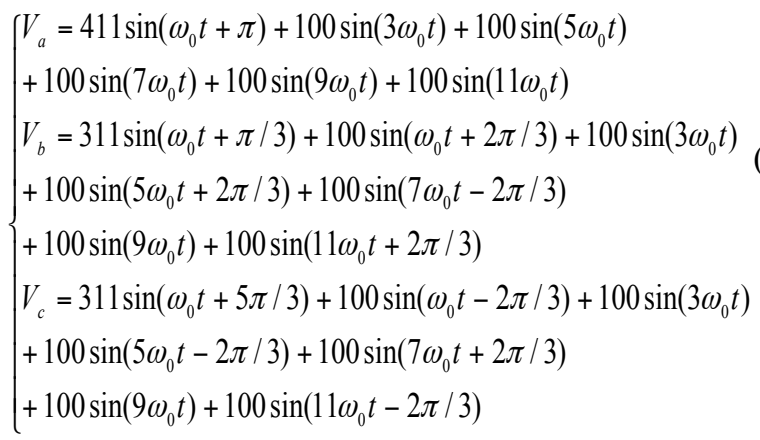

Fig.6 shows dynamic response of the proposed PLL. The three waveforms imply the amplified sinusoidal synchronization signal, the A-phase positive sequence fundamental voltage and the A-phase polluted utility voltage.

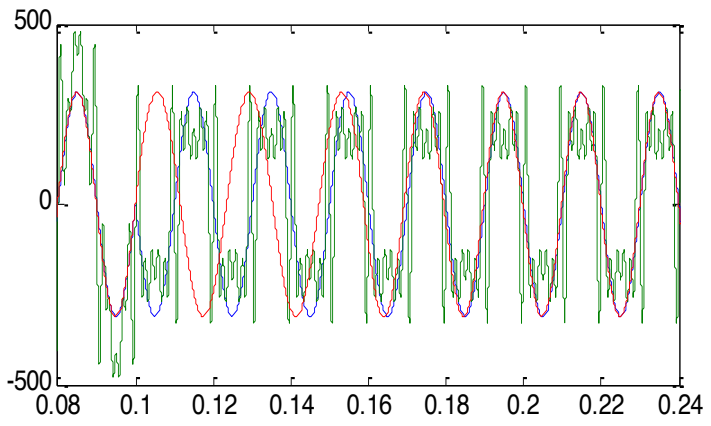

Fig.6 Dynamic response of the proposed PLL when the fundamental phase jumping 


\section{Three-Phase Voltage Unbalance Simulation}

Under the power faulty condition, the three-phase utility voltages with DC offset components and negative sequence component at the fundamental frequency are shown as

$$
\left\{\begin{array}{l}
V_{a}=411 \sin \left(\omega_{0} t\right)+80 \\
V_{b}=311 \sin \left(\omega_{0} t-2 \pi / 3\right)+100 \sin \left(\omega_{0} t+2 \pi / 3\right)+60 \\
V_{c}=311 \sin \left(\omega_{0} t+2 \pi / 3\right)+100 \sin \left(\omega f_{0} t-2 \pi / 3\right)+20
\end{array}\right.
$$

Fig. 7 shows the input and output waveforms of the proposed PLL. The input signals in the presence of unbalance components are described by the waveforms which amplitudes are approximate to $300 \mathrm{~V}$. The other two waveforms stand for the amplified sine and cosine signals of the PLL output. It is not possible to see any significant distortions in the sine and cosine synchronization signals even under severe unbalance conditions. Further, Fig. 8 shows the phase comparison between the A-phase positive sequence fundamental component and the amplified sinusoidal synchronization signal for the proposed PLL method. As shown in Fig.8, the two signals almost go all the way.

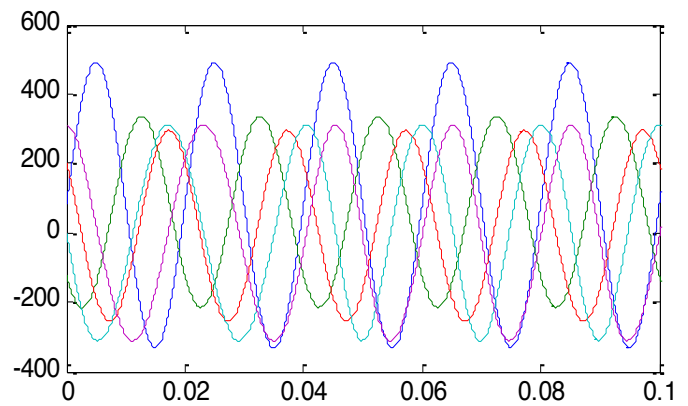

Fig.7 Input and output waveforms of the proposed PLL under the utility voltage unbalance condition

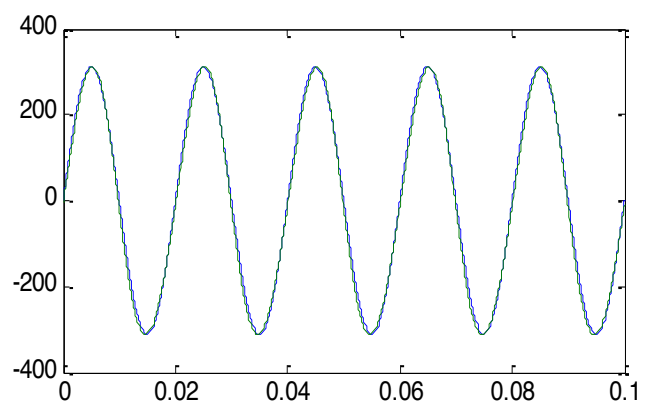

Fig.8 Phase comparison between positive sequence fundamental voltage and amplified sinusoidal synchronization signal for the proposed PLL

\section{CONCLUSIONS}

A new closed-loop phase-locked method for threephase grid-connected inverter or three-phase active PFC has been proposed in this paper. The proposed PLL method is introduced with a formal closed-loop structure including a positive sequence extraction module and a harmonic filter module. The proposed method of PLL is immune to noise, harmonics and other type of distortion. Matlab Digital simulation results show that the proposed
PLL has good steady-state and transient performances even under the conditions of large harmonics, phase shifts and unbalance that exist in the three-phase utility voltages.

\section{REFERENCES}

[1] C. J. Melhorn, T. D. Davis, and G. E. Beam, "Voltage sags: their impact on the utility and industrial customers," IEEE Trans Ind Appl 1998, 34(3): 549-558. http://dx.doi.org/10.1109/28.673725

[2] G. Yaleinkaya, M. H. J. Bollen, and P. A. Crossley, "Characterization of voltage sags in industrial distribution systems," IEEE Trans Ind Appl 1998, 34(4): 682-688. http://dx.doi.org/10.1109/28.703958

[3] B. P. McGrath, D. G. Holmes, and J. J. H. Galloway, "Power converter line synchronization using a discrete fourier transform (DFT) based on a variable sample rate,"IEEE Trans Power Electron 2005, 20(4): 877-884. http://dx.doi.org/10.1109/TPEL.2005.850944

[4] B. Zeng, and Z. Teng, "Parameter estimation of power system signals based on cosine self-convolution window with desirable side-lobe behaviors," IEEE Trans Power Syst 2011, 26(1): 250257. http://dx.doi.org/10.1109/TPWRD.2010.2083701

[5] R. Cardoso, R. F. Camargo, H. Pinheiro, and H. A. Grundling, "Kalman filter based synchronisation methods," IET Gen Transm Distrib 2008, 2(4): 542-555. http://dx.doi.org/10.1049/ietgtd:20070281

[6] P. Rodriguez, A. Luna, R. S. Munoz-Aguilar, I. Etxeberria-Otadui, R. Teodorescu, and F. Blaabjerg, "A stationary reference frame grid synchronization system for three-phase grid-connected power converters under adverse grid conditions," IEEE Trans Power
Electron
2012 ,
27(1): http://dx.doi.org/10.1109/TPEL.2011.2159242

[7] F. Gonzalez-Espin, E. Figueres, and G. Garcera, "An adaptive synchronous reference frame phase-locked loop for power quality improvement in a polluted utility grid," IEEE Trans Ind Electron 2012, 59(6): 2718-2731. http://dx.doi.org/10.1109/TIE.2011.2166236

[8] S. J. Lee, J. K. Kang, and S. K. Sul, "A new phase detecting method for power conversion systems considering distorted conditions in power system," in Proc 34th Annu IEEE Ind Appl Conf 1999, p. 2167-2172.

[9] M. Karimi-Ghartemani and M. R. Iravani, "A method for synchronization of power electronic converters in polluted and variable-frequency environments," IEEE Trans Power Syst 2004, 19(3): 1263-1270. http://dx.doi.org/10.1109/TPWRS.2004.831280

[10] L. G. B. Rolim, D. R. Costa, and M. Aredes, "Analysis and software implementation of a robust synchronizing PLL circuit based on the pq theory ," IEEE Trans Ind Electron 2006, 53(6): 1919-1926. http://dx.doi.org/10.1109/TIE.2006.885483

[11] R. Petrella, A. Revelant, and P. Stocco, "Robust grid synchronization in three-phase distributed power generation systems by synchronous reference frame pre-filtering," in Proc 44th Int Univ Power Eng Conf, 2009, pp. 1-5.

[12] S. Golestan, M. Monfared, F. D. Freijedo, and J. M. Guerrero, "Dynamics assessment of advanced single-phase PLL structures," IEEE Trans Ind Electron 2012, 99: 1-11.

[13] S. Golestan, M. Monfared, F. D. Freijedo, and J. M. Guerrero, "Design and tuning of a modified power-based PLL for singlephase grid connected power conditioning systems," IEEE Trans Power Electron 2012, 27(8): 3639-3650. http://dx.doi.org/10.1109/TPEL.2012.2183894

\section{AUTHORS}

Yingwen Long is with the Shanghai University of Engineering Science, Shanghai, China. (e-mail: longyingwen@sohu.com).

Yuhong Sun is with the Shanghai Surpass Sun Electric Co. Ltd, Shanghai, China. (e-mail: sunyuhong@ssechina.cn).

Submitted 25 May 2015. Published as resubmitted by the author 25 june 2015 . 